\title{
POLE-ON CATACLYSMIC VARIABLES AS Be STARS
}

\author{
R.F. Garrison \\ David Dunlap observatory, University of Toronto \\ Box 360 , Richmond Hill, Ontario, Canada L4C 4 Y 6
}

Cataclysmic-variable stars (CVs) are technically Be stars, since Balmer emission lines appear in their spectra. In general, CV spectra are so unusual that they are easily recognized. The main features are extremely broad, shallow hydrogen lines with broad, faint emission cores. The Balmer emission decrement is very slow, more like that of an H II region than that of a normal Be star. The Balmer decrement in cataclysmic variable stars has been discussed by Elitzur, et a1.

If a CV were viewed pole-on, the spectrum might be quite different. It could exhibit a smooth continuum or may even resemble a normal Be star, except for the Balmer decrement. In this case, there may be a bright cataclysmic variable lurking in the Be star class. A possible example is the star NS 274-67, an 03e star described by Conti et a1. (1986), which has Balmer emission from H-beta to H-epsilon, but no nebular emission at [0 II] or [0 III].

To investigate this possibility in a preliminary way, the list of 1113 southern oB stars from Garrison, Hiltner, and Schild (1977) was re-examined and the Balmer decrements of a 11 Be stars were noted. Several stars, listed in Table 1 , were found to have quite slow decrements.

Even if these "Be stars" are not pole-on CVs, the extreme range of decrements may be worth further investigation. A few may turn out to be due to contamination of the slit by an $H$ II region, but that is not likely to be the case for all of them. The spectra can be examined on the objective prism plates of the Michigan Spectral Atlas to eliminate that possibility.

The idea that pole-on CVs might be found among stars previously classified as Be stars came from a discussion with Brian Warner. The support of the Natural Sciences and Engineering Research Council of Canada is gratefully acknowledged. 


\section{References}

Conti, P., Garmany, C., and Massey, P. 1986, A.J. 92,48. Elitzur, M., Ferland, G., Matthews, W., and Shields, G. 1983 , Ap.J. 272, L 55 .

Garrison, R.F., Hiltner, W.A., and Schild, R.E. 1977, Ap.J.Supp1. 35,111 .

TABLE 1

Be STARS WITH SLOW BALMER DECREMENTS

CPD-48 $1577 \quad 09:$ inne

CPD-44 2597 B2 IVe

HD $83597 \quad$ B 1.5 Ve

HD 85567 B 8 V:ne

HD $94878 \quad$ Bep

CPD-59 3727 B 1:III:nne (not very slow)

HD 105753 B2 IVne (extremely slow)

HD $120678 \quad 08$ III:nep

HD 122669 B I III:e

HD 141926 B2:III:nnep

HD 149313 B2 IV:ne

HD $153295 \quad$ B $1:$ IIIe

HD 154450 B I:IIInne

HD 161103 B 1:III:nne

HD 161774 B 7 nep

HD $163454 \quad$ B 1 IVne

HD 169805 B 1.5 III:nnpe 


\section{DISCUSSION FOLLOWING GARRISON}

Polidan:

In our study of cataclysmic variables with the Voyager UVS we have found that the FUV/UV (912-3200 $\AA$ ) continuum distribution of a cataclysmic variable is quite different from that of an $\mathrm{O}, \mathrm{B}$ or Be star. In fact, the distribution appears to be unique to cataclysmic variables. This is true even for the suspected low inclination systems such as CPD $-48^{\circ} 1577$.

Garrison:

Most of these stars have no FUV photometry. Nor have they been observed for "flickering" on time scales of minutes. I would urge observers to look especially at these stars with flat decrements.

Polidan:

I have your list and we will begin obtaining FUV spectra of these stars as soon as we return to Tucson.

Collins:

Do you know of any "classical" Be star that has ever exploded?

Garrison:

That depends on what you want to call a "classical" Be star: $\eta$ Car? P Cyg? Actually, some of the cataclysmic variables have been quiet for long periods of time. 